Supplement of Earth Surf. Dynam., 6, 595-610, 2018 https://doi.org/10.5194/esurf-6-595-2018-supplement (C) Author(s) 2018. This work is distributed under the Creative Commons Attribution 4.0 License.

(c) (1)

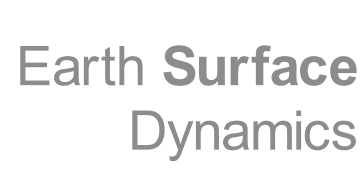

Supplement of

\title{
Tectonic controls of Holocene erosion in a glaciated orogen
}

Byron A. Adams and Todd A. Ehlers

Correspondence to: Byron A. Adams (byron.adams@bristol.ac.uk)

The copyright of individual parts of the supplement might differ from the CC BY 4.0 License. 


\section{This PDF file includes:}

Fig. S1. Basin sample location map.

Fig. S2. Comparison of basin-averaged erosion rates and equilibrium line altitudes (ELA).

Calculation of snow depth estimations.

Fig. S3. Monthly estimated snow depth map.

Fig. S4. Mean monthly snow depth measurements from the Buckinghorse SNOTEL meteorological station.

Table S1. Laboratory and isotopic data for new Olympic Mountain samples.

Table S2. Blank data for new Olympic Mountain samples.

Table S3. Shielding and erosion rate comparisons.

References 


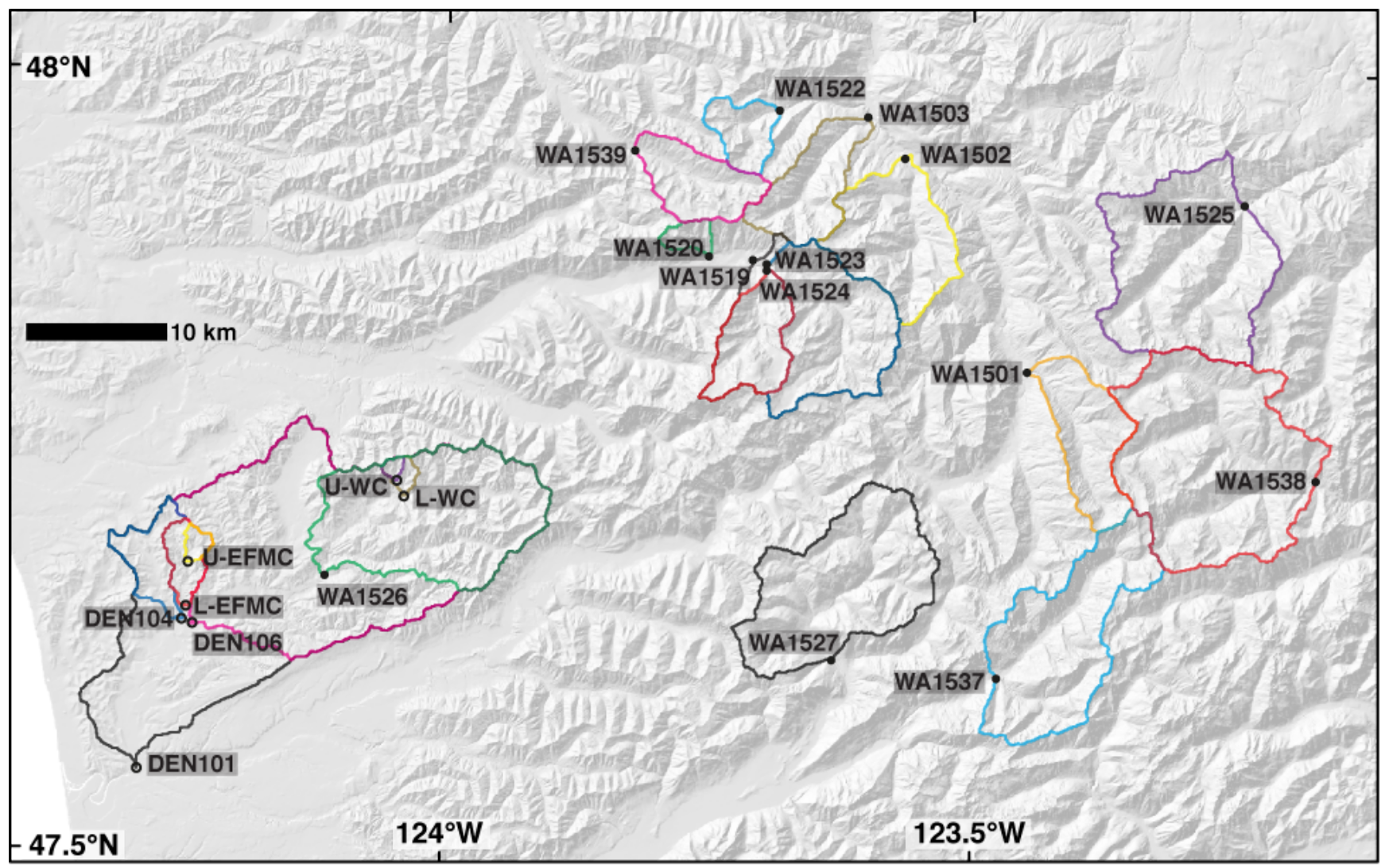

Figure S1. Basin sample location map. Samples marked with open circles are from Belmont et al. (2007).

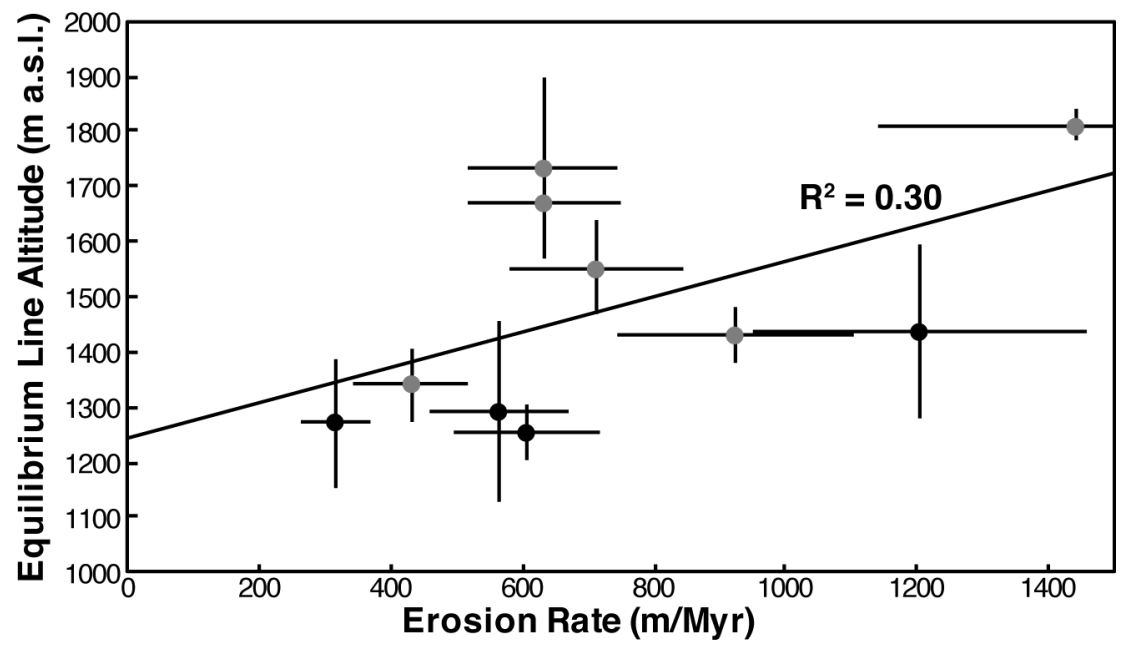

Figure S2. Comparison of basin-averaged erosion rates and equilibrium line altitudes. Black and grey data are from the west and east sides of the range, respectively. 


\section{Calculation of snow depth estimations}

For this study, we utilized the MODIS/Terra Snow Cover Monthly L3 Global 0.05, Version 6 dataset (Hall and Riggs, 2015), to estimate the distribution of snow for a given month. For any given pixel in the scene the value can vary between 0 and 100. While we consider these values to be spatially accurate they are only qualitative in that they do not have a magnitude equal to a physical dimension. To scale these data to represent snow depth values, we consider the snow cover values to be analogous to a percentage of the maximum snow depth in the Olympic Mountain range.

Our best estimate of maximum snow depth in the range comes from the Buckinghorse SNOTEL meteorological station (https://www.ncdc.noaa.gov/cdo-web/datasets/; Network ID: GHCND:USS0023B18S), located at 1484 m a.s.l. in the southern reaches of the Elwha Valley in the core of the range (Figs. S43 and S4). This station records the highest monthly snow depth measurements within the range. In the final step, we smoothed mean monthly snow cover data (2001-2015) using a bilinear algorithm and multiplied these percentages by the mean monthly snow depth station data (2009-2015). We do not assert that the resulting maps (Fig. S3) are completely accurate. However, we have calculated them to constrain the possible effect snow shielding may have on the calculation of erosion rates across the range. We assume a snow density of $0.25 \mathrm{~g} / \mathrm{cm}^{3}$ for shielding calculations. 


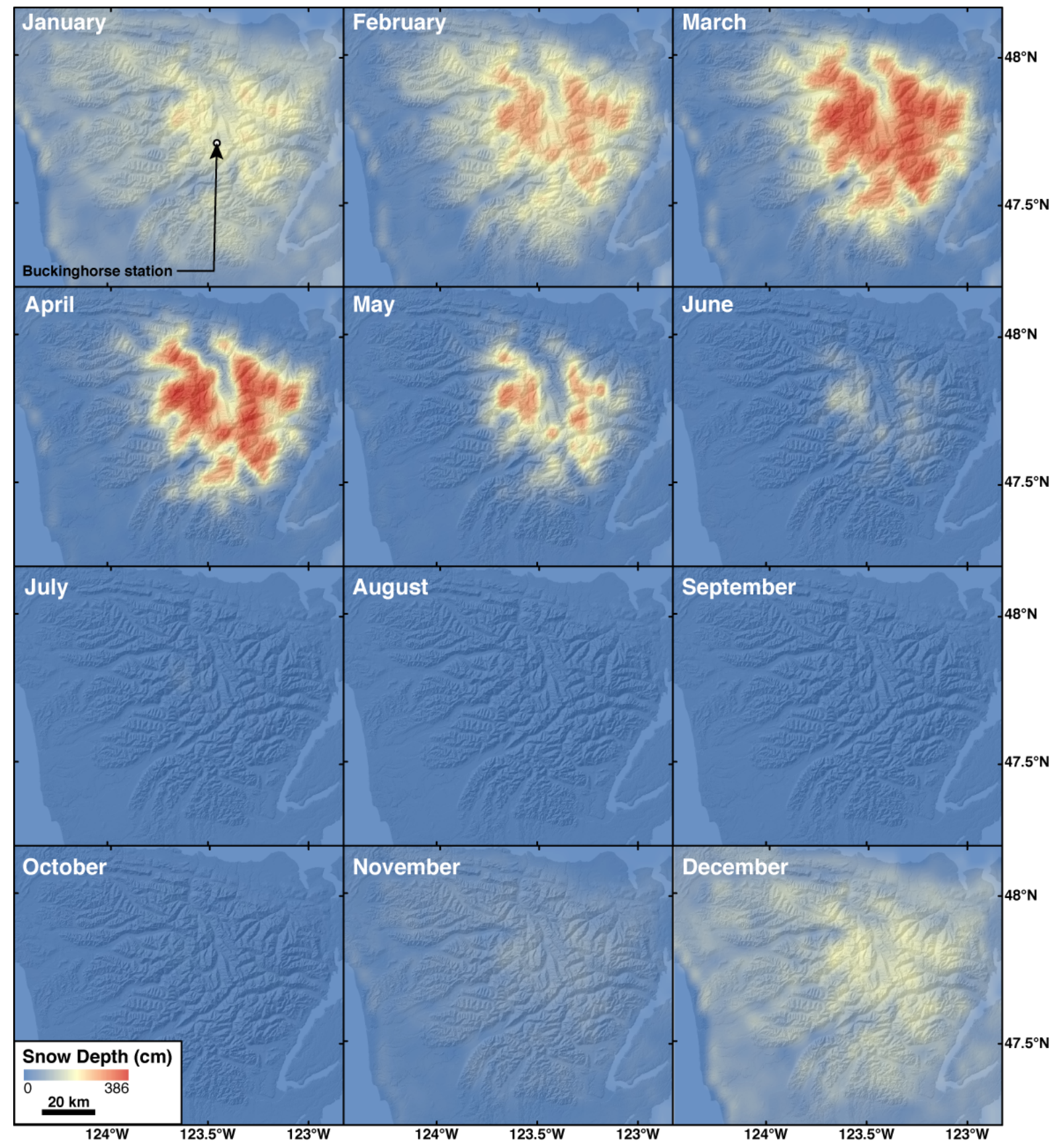

Figure S3. Monthly estimated snow depth map. The dot marks the position of the Buckinghorse SNOTEL meteorological station. 


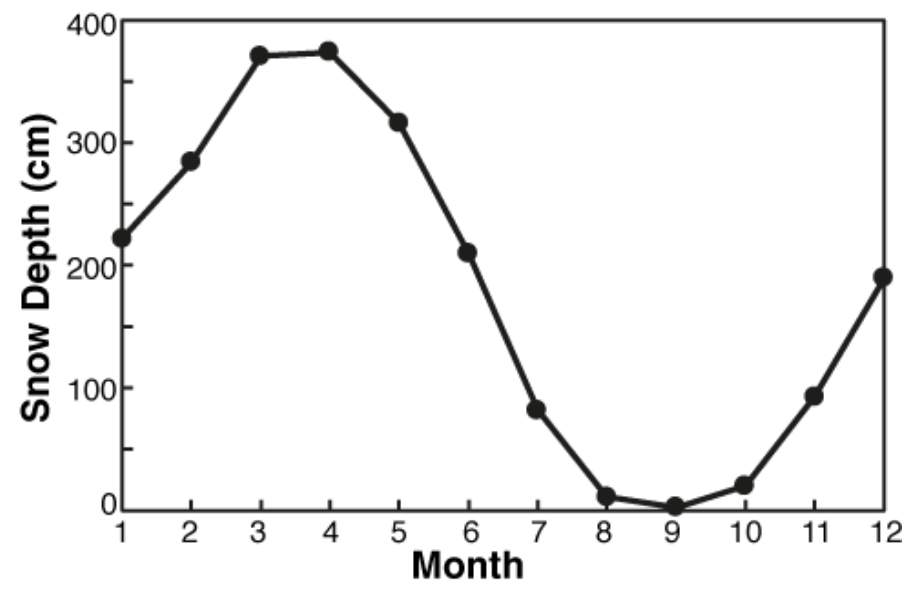

Figure S4. Mean monthly snow depth measurements from the Buckinghorse SNOTEL meteorological station. These means are calculated for the years 2009-2015.

Table S1. Laboratory and isotopic data for new Olympic Mountain samples.

\begin{tabular}{|c|c|c|c|c|c|c|c|c|c|c|c|c|c|c|}
\hline $\begin{array}{c}\text { Sample } \\
\text { Name }\end{array}$ & $\begin{array}{c}\text { Effective } \\
\text { Latitude } \\
\quad\left({ }^{\circ} \mathrm{N}\right)\end{array}$ & $\begin{array}{c}\text { Centroid } \\
\text { Longitude } \\
\left({ }^{\circ} \mathbf{E}\right)\end{array}$ & $\begin{array}{r}\text { Effective } \\
\text { Elevation } \\
\text { (m a.s.l.) }\end{array}$ & $\begin{array}{l}\text { Quartz } \\
\text { (g) }\end{array}$ & $\begin{array}{c}\text { Be from } \\
\text { spike } \\
\text { (g) }\end{array}$ & $\begin{array}{c}\text { Total Al } \\
\text { from ICP } \\
\text { (g) }\end{array}$ & $\begin{array}{l}\text { Laboratory } \\
\text { Be Number }\end{array}$ & $\begin{array}{c}{ }^{10} \mathrm{Be} /{ }^{9} \mathrm{Be} \\
\text { from } \mathrm{AMS}\end{array}$ & $\begin{array}{c}{ }^{10} \mathrm{Be} /{ }^{9} \mathrm{Be} \\
1 \sigma\end{array}$ & $\begin{array}{c}\text { Be Blank } \\
\text { Used }\end{array}$ & $\begin{array}{l}\text { Laboratory } \\
\text { Al Number }\end{array}$ & $\begin{array}{c}{ }^{26} \mathrm{Al}^{27} \mathrm{Al} \\
\text { from AMS }\end{array}$ & $\begin{array}{c}{ }^{26} \mathrm{Al} /{ }^{27} \mathrm{Al} \\
1 \sigma\end{array}$ & $\begin{array}{c}\text { Al Blank } \\
\text { Used }\end{array}$ \\
\hline WA1501 & 47.761893 & -123.38950 & 1397 & 90.382 & $3.29 \mathrm{E}-04$ & $9.2640 \mathrm{E}-03$ & s09728 & $5.0260 \mathrm{E}-14$ & $2.5300 \mathrm{E}-15$ & BA10 & s09935 & $3.257 \mathrm{E}-14$ & $2.850 \mathrm{E}-15$ & BA11 \\
\hline WA 1502 & 47.856058 & -123.57330 & 1266 & 88.882 & $3.29 \mathrm{E}-04$ & $1.8151 \mathrm{E}-02$ & s09729 & $3.9650 \mathrm{E}-14$ & $2.0600 \mathrm{E}-15$ & BA 10 & s09939 & $1.065 \mathrm{E}-14$ & $1.500 \mathrm{E}-15$ & BA11 \\
\hline WA 1503 & 47.96357 & -123.65440 & 1195 & 88.350 & $3.28 \mathrm{E}-04$ & $1.6448 \mathrm{E}-02$ & s09730 & $2.9910 \mathrm{E}-14$ & $1.7300 \mathrm{E}-15$ & BA 10 & s09943 & $9.386 \mathrm{E}-15$ & $1.400 \mathrm{E}-15$ & BA11 \\
\hline WA1519 & 47.86627 & -123.65900 & 1421 & 90.386 & $3.28 \mathrm{E}-04$ & $1.6600 \mathrm{E}-02$ & s09731 & $1.4260 \mathrm{E}-14$ & $1.1200 \mathrm{E}-15$ & BA10 & s09947 & $2.660 \mathrm{E}-15$ & $7.420 \mathrm{E}-16$ & BA11 \\
\hline WA1520 & 47.902426 & -123.77010 & 1184 & 81.778 & $3.27 \mathrm{E}-04$ & $2.0658 \mathrm{E}-02$ & s09732 & $4.2830 \mathrm{E}-14$ & $2.1100 \mathrm{E}-15$ & BA10 & s09951 & $1.303 \mathrm{E}-14$ & $1.670 \mathrm{E}-15$ & BA11 \\
\hline WA1522 & 47.977417 & -123.71920 & 1252 & 51.239 & $3.26 \mathrm{E}-04$ & $6.1441 \mathrm{E}-03$ & s09046 & $3.8700 \mathrm{E}-14$ & $2.5800 \mathrm{E}-15$ & BA1 & s10020 & $5.012 \mathrm{E}-14$ & $3.490 \mathrm{E}-15$ & BA3 \\
\hline WA1523 & 47.89053 & -123.63360 & 1404 & 73.762 & $3.28 \mathrm{E}-04$ & $9.1110 \mathrm{E}-03$ & s09733 & $1.4880 \mathrm{E}-14$ & $1.0900 \mathrm{E}-15$ & BA 10 & s09955 & $3.876 \mathrm{E}-15$ & $8.750 \mathrm{E}-16$ & BA11 \\
\hline WA1524 & 47.832887 & -123.70770 & 1505 & 99.716 & $3.47 \mathrm{E}-04$ & $1.8004 \mathrm{E}-02$ & s09739 & $1.3250 \mathrm{E}-14$ & $1.0000 \mathrm{E}-15$ & BA19 & s09980 & $5.430 \mathrm{E}-15$ & $1.010 \mathrm{E}-15$ & BA20 \\
\hline WA1525 & 47.862877 & -123.29530 & 1547 & 84.053 & $3.27 \mathrm{E}-04$ & $1.0421 \mathrm{E}-02$ & s09735 & $2.3580 \mathrm{E}-14$ & $1.4600 \mathrm{E}-15$ & BA10 & s09967 & $9.652 \mathrm{E}-15$ & $1.450 \mathrm{E}-15$ & BA11 \\
\hline WA1526 & 47.674477 & -124.01220 & 550 & 99.299 & $3.28 \mathrm{E}-04$ & $2.1568 \mathrm{E}-02$ & s09734 & $9.1580 \mathrm{E}-14$ & $3.7000 \mathrm{E}-15$ & BA 10 & s09959 & $2.296 \mathrm{E}-14$ & $2.280 \mathrm{E}-15$ & BA11 \\
\hline WA1527 & 47.616827 & -123.62340 & 1100 & 92.130 & $3.27 \mathrm{E}-04$ & $1.9201 \mathrm{E}-02$ & s09735 & $4.8860 \mathrm{E}-14$ & $2.4200 \mathrm{E}-15$ & BA19 & s09976 & $1.731 \mathrm{E}-14$ & $1.810 \mathrm{E}-15$ & BA20 \\
\hline WA1537 & 47.70025 & -123.41140 & 1147 & 94.016 & $3.48 \mathrm{E}-04$ & $8.3440 \mathrm{E}-03$ & s09740 & $2.2430 \mathrm{E}-14$ & $1.4400 \mathrm{E}-15$ & BA19 & s09984 & $1.722 \mathrm{E}-14$ & $1.920 \mathrm{E}-15$ & $\mathrm{BA} 20$ \\
\hline WA1538 & 47.72078 & -123.27090 & 1439 & 95.680 & $3.48 \mathrm{E}-04$ & $1.0705 \mathrm{E}-02$ & s09741 & $5.0490 \mathrm{E}-14$ & $2.3900 \mathrm{E}-15$ & BA 19 & s09988 & $2.849 \mathrm{E}-14$ & $2.450 \mathrm{E}-15$ & BA20 \\
\hline WA1539 & 47.917853 & -123.76290 & 1238 & 92.504 & $3.48 \mathrm{E}-04$ & $1.4970 \mathrm{E}-02$ & s09742 & $8.6810 \mathrm{E}-14$ & $3.5100 \mathrm{E}-15$ & BA19 & s09992 & $4.037 \mathrm{E}-14$ & $3.120 \mathrm{E}-15$ & BA20 \\
\hline
\end{tabular}

Table S2. Blank data for new Olympic Mountain samples.

\begin{tabular}{|c|c|c|c|c|c|c|c|c|c|c|}
\hline $\begin{array}{l}\text { Blank } \\
\text { Name }\end{array}$ & $\begin{array}{l}\text { Laboratory } \\
\text { Be Number }\end{array}$ & $\begin{array}{c}\text { Be from } \\
\text { spike } \\
\text { (g) }\end{array}$ & $\begin{array}{c}{ }^{10} \mathrm{Be} /{ }^{9} \mathrm{Be} \\
\text { from } \mathrm{AMS}\end{array}$ & $\begin{array}{c}{ }^{10} \mathrm{Be} /{ }^{9} \mathrm{Be} \\
1 \sigma\end{array}$ & $\begin{array}{c}\text { Be } \\
\text { Standard }\end{array}$ & $\begin{array}{l}\text { Laboratory } \\
\text { Al Number }\end{array}$ & $\begin{array}{c}\text { Al from } \\
\text { spike } \\
\text { (g) }\end{array}$ & $\begin{array}{l}{ }^{26} \mathrm{Al} /{ }^{27} \mathrm{Al} \\
\text { from AMS }\end{array}$ & $\begin{array}{c}{ }^{26} \mathrm{Al}^{27} \mathrm{Al} \\
1 \sigma\end{array}$ & $\begin{array}{c}\text { Al } \\
\text { Standard }\end{array}$ \\
\hline BA1 & s09045 & $3.2750 \mathrm{E}-04$ & $1.8410 \mathrm{E}-15$ & $4.9500 \mathrm{E}-16$ & 07KNSTD & -- & -- & -- & - & - \\
\hline BA10 & s09737 & $3.2739 \mathrm{E}-04$ & $1.9620 \mathrm{E}-15$ & $3.6900 \mathrm{E}-16$ & 07KNSTD & -- & -- & -- & - & - \\
\hline BA19 & s09746 & $3.4758 \mathrm{E}-04$ & $2.1870 \mathrm{E}-15$ & $4.2600 \mathrm{E}-16$ & 07KNSTD & -- & -- & -- & - & - \\
\hline BA3 & - & - & - & - & - & s10024 & $2.5761 \mathrm{E}-03$ & $1.647 \mathrm{E}-15$ & $1.028 \mathrm{E}-15$ & KNSTD \\
\hline BA11 & - & -- & - & - & - & s09967 & $2.5512 \mathrm{E}-03$ & $1.905 \mathrm{E}-16$ & $6.079 \mathrm{E}-16$ & KNSTD \\
\hline BA20 & -- & -- & -- & -- & - & s10008 & $2.5582 \mathrm{E}-03$ & $2.077 \mathrm{E}-16$ & $6.176 \mathrm{E}-16$ & KNSTD \\
\hline
\end{tabular}


Table S3. Shielding and erosion rate comparisons.

\begin{tabular}{|c|c|c|c|c|c|c|c|c|}
\hline $\begin{array}{c}\text { Sample } \\
\text { Name }\end{array}$ & $\begin{array}{c}\text { Topographic } \\
\text { Shielding }\end{array}$ & $\begin{array}{l}\text { Snow/Ice } \\
\text { Shielding }\end{array}$ & $\begin{array}{c}\text { Total } \\
\text { Shielding }\end{array}$ & $\begin{array}{c}\text { Topo+Snow } \\
\text { Erosion Rate } \\
(\mathbf{m} / \mathbf{M y r})\end{array}$ & 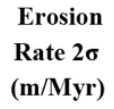 & $\begin{array}{c}\text { Topo Only } \\
\text { Erosion Rate } \\
(\mathbf{m} / \mathbf{M y r})\end{array}$ & $\begin{array}{l}\text { Erosion } \\
\text { Rate } 2 \sigma \\
(\mathrm{m} / \mathrm{Myr})\end{array}$ & $\begin{array}{c}\text { Percent } \\
\text { Difference } \\
(\%)\end{array}$ \\
\hline WA1501 & 0.95 & 0.87 & 0.82 & 638 & 118 & 726 & 136 & 12 \\
\hline WA1502 & 0.96 & 0.85 & 0.80 & 718 & 134 & 842 & 160 & 15 \\
\hline WA1503 & 0.95 & 0.86 & 0.81 & 930 & 183 & 1068 & 212 & 13 \\
\hline WA1519 & 0.95 & 0.85 & 0.80 & 2511 & 618 & 2922 & 725 & 14 \\
\hline WA 1520 & 0.94 & 0.90 & 0.85 & 610 & 112 & 666 & 123 & 8 \\
\hline WA 1522 & 0.95 & 0.87 & 0.82 & 432 & 90 & 492 & 103 & 12 \\
\hline WA1523 & 0.95 & 0.83 & 0.78 & 1881 & 442 & 2238 & 265 & 16 \\
\hline WA1524 & 0.94 & 0.87 & 0.81 & 3117 & 782 & 3558 & 898 & 12 \\
\hline WA 1525 & 0.95 & 0.85 & 0.80 & 1451 & 301 & 1691 & 355 & 14 \\
\hline WA 1526 & 0.97 & 0.96 & 0.92 & 224 & 37 & 235 & 39 & 4 \\
\hline WA 1527 & 0.95 & 0.87 & 0.82 & 564 & 104 & 622 & 115 & 9 \\
\hline WA 1537 & 0.93 & 0.86 & 0.79 & 1213 & 256 & 1396 & 297 & 13 \\
\hline WA1538 & 0.95 & 0.84 & 0.79 & 635 & 116 & 748 & 138 & 15 \\
\hline WA1539 & 0.96 & 0.87 & 0.83 & 318 & 55 & 361 & 63 & 12 \\
\hline U-EFMC & 0.98 & 0.97 & 0.95 & 171 & 34 & 176 & 35 & 2 \\
\hline L-EFMC & 0.98 & 0.97 & 0.96 & 129 & 20 & 131 & 20 & 2 \\
\hline U-WC & 0.97 & 0.96 & 0.93 & 158 & 25 & 164 & 26 & 4 \\
\hline L-WC & 0.98 & 0.96 & 0.93 & 199 & 31 & 207 & 32 & 4 \\
\hline DEN104 & 0.99 & 0.97 & 0.96 & 114 & 43 & 117 & 44 & 2 \\
\hline DEN106 & 0.97 & 0.96 & 0.93 & 237 & 110 & 246 & 114 & 3 \\
\hline DEN101 & 0.98 & 0.96 & 0.94 & 223 & 176 & 231 & 182 & 3 \\
\hline
\end{tabular}

\section{References}

Belmont, P., Pazzaglia, F., and Gosse, J. C.: Cosmogenic 10 Be as a tracer for hillslope and channel sediment dynamics in the Clearwater River, western Washington State, Earth and Planetary Science Letters, 264, 123-135, 2007.

Hall, D., and Riggs, G.: MODIS/Terra snow cover Monthly L3 Global 0.05Deg CMG, Version 6. In: NASA National Snow and Ice Data Center, Distributed Active Center, Boulder, Colorado, USA, 2015. 